\title{
COMPARAÇÃO DE DIFERENTES METODOLOGIAS PARA ESTIMATIVA DE CURVAS INTENSIDADE-DURAÇÃO-FREQÜÊNCIA PARA PELOTAS - RS
}

\author{
RITA DE C. F. DAMÉ ${ }^{1}$, CLAUDIA F. A. TEIXEIRA ${ }^{1}$, VIVIANE S. S. TERRA ${ }^{2}$
}

\begin{abstract}
RESUMO: Nos projetos agrícolas de obras hidráulicas, onde não se dispõe de dados observados de vazão, é necessário explorar ao máximo as informações relativas às curvas Intensidade-DuraçãoFreqüência (IDF). Diante disso, é preciso obter maneira de desenvolver metodologias de estimativas de curvas IDF, em locais que possuam pouco ou nenhum dado pluviográfico. O objetivo do trabalho foi comparar as metodologias de desagregação de precipitações diárias para verificar o ganho de informação em termos de curvas IDF, comparadas àquela obtida a partir de dados observados (histórica). Os métodos utilizados foram: (a) Método das Relações (CETESB, 1979); (b) BELTRAME et al. (1991); (c) ROBAINA \& PEITER (1992); (d) Modelo Bartlett-Lewis do Pulso Retangular Modificado (DAMÉ, 2001). Utilizou-se de série de dados de precipitação diária de Pelotas - RS, referente ao período de 1982-1998. Para estimar as curvas IDF, a partir dos registros históricos, foram estabelecidas as durações de $15 ; 30 ; 60 ; 360 ; 720$ e 1.440 minutos, e os períodos de retorno de 2; 5 e 10 anos. Os valores de intensidades máximas foram comparados entre si, pelo teste " $t$ " de Student, para os coeficientes linear e angular, e pelo Erro Relativo Médio Quadrático. O método que melhor representou as intensidades máximas de precipitação, nos períodos de retorno de 2 e 10 anos, foi o Método das Relações (CETESB, 1979).
\end{abstract}

PALAVRAS-CHAVE: precipitação máxima, método das relações, desagregação de chuva diária.

\section{COMPARISON OF DIFFERENT METHODOLOGIES TO ESTIMATE INTENSITY- DURATION-FREQUENCY CURVES FOR PELOTAS - RS, BRAZIL}

\begin{abstract}
Agricultural projects which deal with hydraulic projects and do not possess observed data on outflow need to explore at the most, information about the Intensity-Duration-Frequency (IDF) curves. Thus, it is necessary to create ways to develop methodologies that estimate IDF curves for locations that have little or no pluviometric data. The aim of this work was to compare disaggregation methodologies for daily precipitation, to verify the increase in quality information considering the IDF curves, as compared to those originated from observed data (historical records). Four methods were tested: (a) Method of Relations (CETESB, 1979); (b) one proposed by BELTRAME et al. (1991); (c) one proposed by ROBAINA \& PEITER (1992), and (c) the BartlettLewis Model of Modified Rectangular Pulse (DAMÉ, 2001). A data set for daily precipitation in Pelotas-RS for the period of 1982-1998 was used, and to estimate IDF curves through historical records six time periods were established: $15 ; 30 ; 60 ; 360 ; 720$ and 1,440 minutes, together with return periods of 2; 5 and 10 years. Values for maximum intensities were compared through the Student " $\mathrm{t}$ " test for the linear and angular coefficients, and through the relative mean square error. The method that best simulated maximum precipitation intensity for the return periods of 2 and 10 years was the Method of Relations (CETESB, 1979).
\end{abstract}

KEYWORDS: maximum precipitation, relationship method, daily rain disaggregation.

\footnotetext{
${ }^{1}$ Eng ${ }^{\mathrm{a}}$ Agrícola, Profa. Doutora, Departamento de Engenharia Agrícola, UFPel, Pelotas - RS, Fone: (0XX53) 3275.7315, ritah2o@hotmail.com

${ }^{2}$ Eng ${ }^{\underline{a}}$ Agrícola, Pós-Graduanda em Gestão Regional de Recursos Hídricos, Departamento de Engenharia Agrícola, UFPel, Pelotas RS. 


\section{INTRODUÇÃO}

As chuvas constituem-se na principal entrada de água em uma bacia hidrográfica, e a sua quantificação, bem como o conhecimento da forma como se distribui temporal e espacialmente são fundamentais em estudos relacionados à necessidade de irrigação, disponibilidade de água para abastecimento doméstico e industrial, erosão do solo, controle de inundações, entre outros.

Para a caracterização das precipitações, é necessário conhecer a sua duração, sua intensidade e sua freqüência de ocorrência ou período de retorno (Tr). Essa relação é comumente denominada de curvas Intensidade-Duração-Freqüência de ocorrência (IDF), sendo uma ferramenta utilizada nos processos de transformação chuva-vazão.

Nos projetos agrícolas de obras hidráulicas, onde não se dispõe de dados observados de vazão, é necessário explorar ao máximo as informações relativas à precipitação (IDF), bem como ao sistema físico (bacia hidrográfica) para a obtenção da vazão de projeto. Entretanto, os dados pluviográficos não são tão facilmente disponíveis; o que existe em abundância, são dados pluviométricos. Diante dessa realidade, é preciso desenvolver metodologias de estimativas de curvas IDF, em locais que possuam pouco ou nenhum dado pluviográfico.

Para isso, uma das alternativas possíveis é utilizar uma série de dados de precipitação máxima diária anual do local onde será realizado o estudo hidrológico, que seja representativa, estacionária e homogênea (BRUSA, 2004) para, a partir dessa, obter alturas de chuva com intervalos de tempo subdiários (DAMÉ, 2001). Obtendo-se dados de precipitação diária, associados aos períodos de retorno de interesse, pode-se utilizar metodologia de desagregação adequada. Assim, a relação entre as alturas de precipitação, em função da duração de desagregação e o período de retorno, originam curvas IDF a partir de registros pluviométricos.

A técnica de desagregação, que é de uso corrente na prática da Engenharia, baseia-se nos coeficientes de desagregação (CETESB, 1979). Ainda na forma analítica, encontra-se o trabalho de ROBAINA \& PEITER (1992), cujo objetivo foi testar o desempenho de um modelo de desagregação de chuvas intensas, com a finalidade de gerar precipitações máximas médias em durações inferiores a 24 horas. Existem, ainda, metodologias que envolvem a simulação da precipitação em duração sub-horária, e a associação da série simulada a modelos estocásticos de desagregação (RODRIGUEZ-ITURBE et al., 1987; KOUTSOYIANNIS \& XANTHOPOULOS, 1990; GLASBEY et al., 1995; DAMÉ, 2001), possibilitando a obtenção das curvas IDF.

No meio científico, tem-se buscado um modelo de desagregação de chuva diária que leve a obter curvas IDF cujo desvio, comparado às históricas, seja admissível de ser utilizado em projetos de obras hidráulicas, ou seja, na estimativa da chuva de projeto, que é um dos dados de entrada em modelos que fazem a transformação chuva-vazão.

Sendo assim, a hipótese do presente trabalho é avaliar se não há diferença significativa entre os valores de intensidades máximas obtidas a partir de dados históricos e aqueles obtidos por desagregação de chuva diária.

O objetivo do presente trabalho foi avaliar o ganho de informação em termos de curvas Intensidade-Duração-Freqüência de ocorrência (IDF), quando utilizados modelos de desagregação de chuva diária para a obtenção dessas. Para tanto, foi utilizada a curva IDF histórica do município de Pelotas - RS, e as seguintes metodologias: a) método das relações (CETESB, 1979); (b) BELTRAME et al. (1991); (c) ROBAINA \& PEITER (1992), e (d) modelo Bartlett-Lewis do Pulso Retangular Modificado (DAMÉ, 2001).

\section{MATERIAL E MÉTODOS}

A estimativa das curvas IDF históricas foi realizada utilizando-se de série de 17 anos de registros pluviográficos de Pelotas - RS (1982 a 1998), obtidos da Estação Agroclimatológica de Pelotas - Convênio EMBRAPA/UFPel, INMET (DAMÉ, 2001). Para a obtenção das duas curvas 
IDF, a histórica e a sintética (obtidas pelos métodos de desagregação de chuva diária), o período temporal foi o mesmo.

Para estimar as curvas IDF, a partir dos registros históricos, foram estabelecidas as durações de $15 ; 30 ; 60 ; 360 ; 720$ e 1.440 minutos e os períodos de retorno de $2 ; 5$ e 10 anos. Assim, após a leitura dos pluviogramas, a partir do software GEDAC - Gerenciamento de Dados Contínuos (PEDROLLO, 1997), foram constituídas as séries de intensidades máximas diárias anuais, nas durações preestabelecidas e, mediante a equação de plotagem de Weibull (LANNA, 2001), foi obtida a freqüência de ocorrência dos valores de intensidades máximas para cada duração.

Para a aplicação das metodologias de desagregação de CETESB (1979), BELTRAME et al. (1991) e ROBAINA \& PEITER (1992), foi necessário selecionar um modelo teórico de probabilidade. Para tanto, usou-se o software WINSTAT (MACHADO \& CONCEIÇÃO, 2006), considerando a estimativa dos parâmetros pelo método da máxima verossimilhança e a seleção do modelo pelo papel de probabilidade. A partir do modelo probabilístico selecionado, foram estimadas as precipitações máximas diárias, para os períodos de retorno previamente estabelecidos.

Para desagregar a chuva diária mediante o método das relações (CETESB, 1979), as precipitações máximas diárias, para os períodos de retorno estabelecidos, foram multiplicados pelos coeficientes apresentados na Tabela 1, o que permitiu gerar pontos suficientes para definir as curvas IDF, a partir de registros pluviométricos.

TABELA 1. Coeficientes de desagregação de dados pluviométricos (CETESB, 1979). Disaggregation coefficients for the pluviometric data (CETESB, 1979).

\begin{tabular}{ccccccc}
\hline $\begin{array}{c}\text { Relação de } \\
\text { Duração }\end{array}$ & $24 \mathrm{~h}^{*} / 1$ dia**$^{* *}$ & $12 \mathrm{~h} / 24 \mathrm{~h}$ & $10 \mathrm{~h} / 24 \mathrm{~h}$ & $8 \mathrm{~h} / 24 \mathrm{~h}$ & $6 \mathrm{~h} / 24 \mathrm{~h}$ & $1 \mathrm{~h} / 24 \mathrm{~h}$ \\
\hline Coeficiente & 1,14 & 0,85 & 0,82 & 0,78 & 0,72 & 0,42 \\
\hline $\begin{array}{c}\text { Relação de } \\
\text { Duração }\end{array}$ & $30 \mathrm{~min} / 1 \mathrm{~h}$ & $25 \mathrm{~min} / 1 \mathrm{~h}$ & $20 \mathrm{~min} / 1 \mathrm{~h}$ & $15 \mathrm{~min} / 1 \mathrm{~h}$ & $10 \mathrm{~min} / 1 \mathrm{~h}$ & $5 \mathrm{~min} / 1 \mathrm{~h}$ \\
\hline Coeficiente & 0,74 & 0,91 & 0,81 & 0,70 & 0,54 & 0,34 \\
\hline
\end{tabular}

* maior valor de precipitação correspondente ao período consecutivo de 24 horas.

** valor compreendido entre os horários de precipitação pluviométrica.

A metodologia empregada por BELTRAME et al. (1991) parte do pressuposto da existência de duas relações que tenham validade regional ou sub-regional. A primeira relação permite a estimativa dos valores adimensionais com o tempo de retorno $\mathrm{Tr}$, dos totais precipitados com qualquer duração $\mathrm{d}$, notados por $\mathrm{X}(\mathrm{Tr}, \mathrm{d}) / \mathrm{Xm}(\mathrm{d})$. A segunda relação permite a estimativa da média dos totais precipitados em determinada duração de chuva, $\mathrm{Xm}(\mathrm{d})$, como função dessa duração. $\mathrm{O}$ produto do fator $\mathrm{X}(\mathrm{Tr}, \mathrm{d}) / \mathrm{Xm}(\mathrm{d})$ por $\mathrm{Xm}(\mathrm{d})$ fornece a altura máxima precipitada para um dado período de retorno e uma dada duração. Os valores de intensidade máxima para uma dada duração e período de retorno I $(\mathrm{Tr}, \mathrm{d})$ são obtidos dividindo-se $\mathrm{X}(\mathrm{Tr}, \mathrm{d})$ por $\mathrm{d}$.

O método proposto por ROBAINA \& PEITER (1992) supõe que exista uma expressão que defina matematicamente a relação entre o total precipitado em um tempo de duração qualquer e o total precipitado no período de 24 horas [eq.(1)]:

$$
\mathrm{P}\left(\mathrm{t}, \mathrm{T}_{\mathrm{r}}\right)=\mathrm{P}(24) \mathrm{F}(\mathrm{t}) \mathrm{F}\left(\mathrm{T}_{\mathrm{r}}\right)
$$

em que,

$\mathrm{P}(\mathrm{t}, \mathrm{Tr})$ - lâmina total precipitada de duração e período de retorno preestabelecidos;

$\mathrm{P}(24)$ - lâmina precipitada média com duração de 24 horas;

$\mathrm{F}(\mathrm{t})$ - função de desagregação, e

$\mathrm{F}(\mathrm{Tr})$ - função do período de retorno.

As funções de desagregação e do período de retorno são obtidas pelas seguintes equações: 


$$
\mathrm{F}(\mathrm{t})=0,0000768 \mathrm{t}+0,1396 \ln (1+0,3333 \mathrm{t})
$$

Nessa expressão, o tempo de duração deve ser expresso em minutos:

$$
\mathrm{F}\left(\mathrm{T}_{\mathrm{r}}\right)=0,4297^{\mathrm{m}} \mathrm{T}_{\mathrm{r}}^{\mathrm{m}}
$$

O expoente m é função da duração da precipitação e é obtido pela seguinte expressão:

$$
\mathrm{m}=\frac{0,2086 \mathrm{t}}{(\mathrm{t}+4,5969)}
$$

No método usado por DAMÉ (2001), o autor ajustou os parâmetros do modelo Bartlett-Lewis do Pulso Retangular Modificado (BLPRM) (RODRIGUEZ-ITURBE, 1987) e, com a resposta desse, as séries de precipitação na duração de 15 minutos foram agregadas à duração de 24 horas e usadas no modelo de desagregação proposto por GLASBEY et al. (1995).

O modelo BLPRM supõe que o processo de precipitação tenha estacionaridade mensal, ou seja, que suas características estatísticas (média, variância, co-variância, probabilidade de ocorrência de períodos secos) não variem dentro do mês. Logo, a precipitação foi simulada para cada um dos 12 meses do ano.

Para iniciar o processo de simulação, os meses do ano foram divididos em intervalos de tempo de 15 minutos $(n \Delta t)$, em que " $n$ " é o número de intervalos de tempo $(\Delta t)$ de 15 minutos existente em cada mês. Para os meses com 31 dias, o valor de $n=2.976$, para 30 dias $n=2.880$, para 29 dias $\mathrm{n}=2.784$ e para 28 dias $\mathrm{n}=2.688$.

$\mathrm{O}$ procedimento de simulação obedeceu à seguinte ordem: primeiro foram simulados os tempos de início dos eventos de precipitação $\left(\mathrm{TIT}_{\mathrm{i}}\right.$ ); após, os tempos de início das células de precipitações (TIOC) $)_{i, j}$, associadas a cada um dos eventos, e, para cada uma dessas células, foram simuladas as suas durações $\left(\mathrm{D}_{\mathrm{i}, \mathrm{j}}\right)$ e respectivas intensidades $\left(\mathrm{I}_{\mathrm{i}, \mathrm{j}}\right)$.

\section{Simulação dos tempos de início dos eventos de precipitação $\left(\right.$ TIT $\left._{\mathbf{i}}\right)$}

Para simular o tempo de início dos eventos $\left(\mathrm{TIT}_{\mathrm{i}}\right)$, dentro de cada mês, foram gerados valores pseudo-aleatórios com distribuição exponencial, a partir de valores com distribuição uniforme entre 0 e 1 , conforme eq.(5):

$$
\operatorname{TIT}_{\mathrm{i}}=\frac{\operatorname{Ln}(\mathrm{u})}{-\lambda}+\operatorname{TIT}_{(\mathrm{i}-1)}
$$

em que,

$\operatorname{TIT}_{\mathrm{i}}$ - tempo de início do evento $\mathrm{i}(\Delta \mathrm{t})$, para $\operatorname{TIT}_{\mathrm{i}} \leq(\mathrm{n} \Delta \mathrm{t})$;

i - evento $1,2,3 \ldots . . . z$;

$\mathrm{u}$ - valores com distribuição uniforme entre 0 e 1, e

$\lambda$ - parâmetro do modelo BLPRM que governa o tempo de origem dos eventos, $\Delta \mathrm{t}^{-1}$.

Cada valor de $\mathrm{TIT}_{\mathrm{i}}$ simulado foi comparado ao $(\mathrm{n} \Delta \mathrm{t})$ do mês. O processo de simulação do TIT $_{\mathrm{i}}$ terminou quando foi simulado um valor de TIT $_{\mathrm{i}}>(\mathrm{n} \Delta \mathrm{t})$. O significado de um valor de TIT $_{\mathrm{i}}>$ $(n \Delta t)$ é que o evento não pertence ao mês no qual está sendo simulada a precipitação. Na simulação do primeiro valor de TIT $_{\mathrm{i}}$, o valor de $\mathrm{TIT}_{(\mathrm{i}-1)}$ é nulo.

\section{Simulação dos tempos de início das células de precipitação (TIOC) $)_{i, j}$}

Os tempos em que ocorrem as células de precipitação associadas aos eventos $\left(\mathrm{TIOC}_{\mathrm{i}, \mathrm{j}}\right)$ são variáveis aleatórias exponencialmente distribuídas, assim como os TIT $_{\mathrm{i}}$. Logo, a expressão usada na simulação de (TIOC) $)_{i, j}$ foi a eq.(6): 


$$
(\mathrm{TIOC})_{i, j}=\frac{\operatorname{Ln}(\mathrm{u})}{-\kappa \eta_{i}}+(\text { TIOC })_{i, j-1}
$$

em que,

$(\mathrm{TIOC})_{\mathrm{i}, \mathrm{j}}$ - tempo de início de origem de células de precipitação “ $\mathrm{j}$ ”, associadas aos eventos "i" $(\Delta \mathrm{t})$;

$\mathrm{i}$ - evento $1,2,3 \ldots . . . \mathrm{z}$;

j - célula de precipitação $1,2,3 \ldots y$, associada ao evento i;

$\kappa$ - parâmetro do modelo BLPRM responsável pela simulação do (TIOC) $)_{\mathrm{i}, \mathrm{j}}$, adimensional;

$\eta_{\mathrm{i}}$ - variável aleatória associada a cada evento $\left(\Delta \mathrm{t}^{-1}\right)$, com distribuição gama, de índice $\alpha \mathrm{e}$ parâmetro de escala $v(\Delta t)$, cuja esperança matemática e variância são representadas, respectivamente, por $\mathrm{E}[\eta]=\alpha / \nu$ e $\operatorname{VAR}[\eta]=\alpha / v^{2}$. No BLPRM, há um valor de $\eta$ associado a cada evento de precipitação $\left(\eta_{\mathrm{i}}\right)$.

$\mathrm{O}$ valor de $\Delta \mathrm{t}$, no qual ocorreu a primeira célula de precipitação (TIOC) $)_{\mathrm{i}, 1}$, foi o mesmo do $\mathrm{TIT}_{\mathrm{i}}$, pois o modelo BLPRM supõe que essa célula tenha origem com o início do evento. $\mathrm{O}$ posicionamento temporal das demais células de precipitação (TIOC) $)_{i, 2},(\text { TIOC })_{i, 3}, \ldots . . .(\text { TIOC })_{i, y}$, foi determinado por meio da eq.(7), na qual, após um tempo exponencialmente distribuído, com média $1 /(\phi \eta)(\Delta \mathrm{t})$, nenhuma célula mais se originou.

Assim, a cada simulação, o valor de (TIOC) $)_{\mathrm{i}, \mathrm{j}}$ foi comparado ao tempo de fim de origem de células $(\mathrm{TFOC})_{\mathrm{i}}$. O processo de simulação de (TIOC) $)_{\mathrm{i}, \mathrm{j}}$ terminou quando esse valor foi maior que $(\mathrm{TFOC})$.

A eq.(7) indica a forma de estimar o (TFOC) $)_{\mathrm{i}}$ :

$$
(\mathrm{TFOC})_{\mathrm{i}}=\frac{\operatorname{Ln}(\mathrm{u})}{-\phi \eta}+\text { TIT }_{\mathrm{i}}
$$

em que,

i - evento $1,2,3 \ldots . . . z$

$(\mathrm{TFOC})_{\mathrm{i}}$ - tempo de fim de origem de células de precipitação, as quais estão associadas ao evento i, $\Delta \mathrm{t}$, e

$\phi$ - parâmetro do modelo BLPRM, responsável pela simulação do (TFOC) ${ }_{\mathrm{i}}$, adimensional.

\section{Simulação da duração e intensidade das células de precipitação}

A duração das células de precipitação, associada a cada evento, é considerada uma variável aleatória com distribuição exponencial, cuja média é $1 / \eta(\Delta t)$. Para simular a duração de cada uma dessas células, foram gerados valores pseudo-aleatórios com distribuição exponencial, a partir de valores com distribuição uniforme entre 0 e 1 , conforme eq.(8):

$$
\mathrm{D}_{\mathrm{i}, \mathrm{j}}=\frac{\operatorname{Ln}(\mathrm{u})}{-\eta_{\mathrm{i}}}
$$

em que,

$\mathrm{D}_{\mathrm{i}, \mathrm{j}}$ - duração da célula de precipitação “j”, associada ao evento “i”, $\Delta \mathrm{t}$;

$\mathrm{i}$ - evento $1,2,3 \ldots \ldots . \mathrm{z}$, e

j - célula de precipitação $1,2,3 \ldots . . . \mathrm{y}$, associada ao evento i.

As intensidades das células também são consideradas variáveis aleatórias, com distribuição exponencial, e seus valores foram obtidos conforme eq.(9):

$$
\mathrm{I}_{\mathrm{i}, \mathrm{j}}=\frac{\operatorname{Ln}(\mathrm{u})}{-1 / \mu_{\mathrm{X}}}
$$


em que,

$\mathrm{I}_{\mathrm{i}, \mathrm{j}}$ - intensidade da célula de precipitação “j”, associada ao evento “i”, $\mathrm{mm} \Delta \mathrm{t}^{-1}, \mathrm{e}$

$\mu_{\mathrm{x}}$ - parâmetro do modelo BLPRM, que representa a média das intensidades das células de precipitação associadas aos eventos, $\mathrm{mm} \Delta \mathrm{t}^{-1}$.

Uma vez simulada a ocorrência, a intensidade e a duração das células de precipitação em $\Delta \mathrm{t}=$ 15 minutos, a intensidade de cada evento foi obtida somando-se a intensidade de cada uma das células de precipitação. A precipitação total mensal foi obtida somando-se o total precipitado em cada um dos eventos ocorridos dentro do mês.

Depois de simulada a precipitação na duração de 15 minutos, esses valores foram agregados na duração de 24 horas. Tal procedimento foi feito porque as séries de precipitação, nas durações de 15 minutos e 24 horas, foram usadas na desagregação da precipitação diária.

Uma vez apresentada a estrutura de simulação do modelo BLPRM, o próximo passo foi ajustar os parâmetros do modelo. Conforme apresentado, os seus parâmetros são $\lambda, v, \kappa, \mu_{\mathrm{x}}, \alpha, \phi$. $\mathrm{O}$ parâmetro $\lambda\left(\Delta \mathrm{t}^{-1}\right)$ governa o tempo de origem dos eventos; $\kappa$ (adimensional) é responsável pela simulação do tempo de origem das células de precipitação associadas aos eventos; $\phi$ (adimensional) é usado na simulação do tempo em que não mais se originam células de precipitação; $\mu_{\mathrm{x}}\left(\mathrm{mm} \Delta \mathrm{t}^{-1}\right)$ representa a média das alturas de células de precipitação associadas aos eventos, e $\alpha$ (adimensional) e $v(\Delta t)$ são parâmetros de forma e escala da distribuição gama que, na simulação da precipitação por meio desse modelo, é usada para simular $\eta\left(\Delta \mathrm{t}^{-1}\right)$, o qual auxilia na estimativa da duração das células de precipitação.

De posse dos dados desagregados de precipitação $(\mathrm{mm})$, na duração de 15 minutos, esses foram agregados para as durações preestabelecidas. Essas alturas de precipitação $(\mathrm{mm})$ foram transformadas em intensidades $\left(\mathrm{mm} \mathrm{h}^{-1}\right)$ e, a partir dessas, obtidas as curvas IDF sintéticas.

Uma vez aplicadas as metodologias supracitadas, foi necessário avaliar se há ou não diferença significativa, em nível $\alpha$ de probabilidade, entre os valores de intensidades máximas históricas e os obtidos mediante os métodos de desagregação da precipitação diária. Para tanto, foram utilizadas as metodologias do teste " $\mathrm{t}$ " de Student com n-k graus de liberdade, sendo "n" o tamanho da amostra e " $k$ " o número de variáveis explicativas para os coeficientes linear e angular, e a metodologia do Erro Relativo Médio Quadrático (RMS).

A partir do teste "t" de Student, foi testada a hipótese de nulidade $\left(\mathrm{H}_{0}\right)$ para os coeficientes linear $\left(\beta_{0}\right)$ e angular $\left(\beta_{1}\right)$. Assim, se a estatística do teste " $t$ ", para ambos os coeficientes, for inferior aos valores de "t" tabelado para um nível de probabilidade (1- $\alpha$ ), sendo $\alpha=5 \%$, aceita-se $\mathrm{H}_{0}$ e entende-se que não há diferença significativa entre os valores de intensidades máximas históricas e os valores de intensidades máximas, obtidas mediante os modelos de desagregação. Também foram avaliados os níveis de significância dos coeficientes linear $\left(\beta_{0}\right)$ e angular $\left(\beta_{1}\right)$, aceitando-se $\mathrm{H}_{0}$, quando os valores calculados de significância, para ambos os coeficientes, forem superiores a $5 \%$ de probabilidade. Os coeficientes $\beta_{0}$ e $\beta_{1}$ do modelo linear são apresentados na eq.(10), sendo $X$ os valores de intensidades máximas históricos nas diversas durações e períodos de retorno, e Y os valores obtidos mediante a metodologia de desagregação.

$$
\mathrm{Y}=\beta_{0}+\beta_{1} \mathrm{X}
$$

As hipóteses a serem testadas são:

$$
\begin{array}{lll}
\mathrm{H}_{\mathrm{o}}: \beta_{0}=0 & \mathrm{H} & \mathrm{H}_{\mathrm{o}}: \beta_{1}=1 \\
\mathrm{H}_{1}: \beta_{0} \neq 0 & & \mathrm{H}_{1}: \beta_{1} \neq 1
\end{array}
$$

A estatística do teste é: 


$$
\mathrm{t}=\frac{\hat{\beta}_{\mathrm{i}}-\beta_{\mathrm{i}}}{\sigma\left(\hat{\beta}_{\mathrm{i}}\right)}
$$

Outra metodologia aplicada foi a estimativa do Erro Relativo Médio Quadrático (RMS), que fornece indicação do grau de precisão dos modelos de desagregação testados, conforme eq.(12):

$$
\mathrm{RMS}=\sqrt{\frac{\sum_{\mathrm{i}=1}^{\mathrm{n}}\left(\frac{\mathrm{I}_{\mathrm{s}}-\mathrm{I}_{\mathrm{h}}}{\mathrm{I}_{\mathrm{h}}}\right)^{2}}{\mathrm{n}}}
$$

em que,

$\mathrm{I}_{\mathrm{s}}$ - valores de intensidades máximas sintéticos $\left(\mathrm{mm} \mathrm{h}^{-1}\right)$, ocorridos na duração escolhida "D" (min) e período de retorno "Tr", anos;

$\mathrm{I}_{\mathrm{h}}$ - valores de intensidades máximas históricos $\left(\mathrm{mm} \mathrm{h}^{-1}\right)$, ocorridos na duração escolhida " $\mathrm{D}$ " (min) e período de retorno "Tr", anos, e

$\mathrm{n}$ - número de durações utilizadas no estabelecimento das curvas IDF.

\section{RESULTADOS E DISCUSSÃO}

A partir das precipitações do município de Pelotas - RS, no período de 1982 a 1998, foi obtida a série de precipitação máxima diária anual, da qual foram estimados os parâmetros do modelo probabilístico Gumbel - Extremos Tipo I, por ter sido esse o que melhor representou o conjunto de dados amostrais, visto a aderência dos dados históricos à reta (Figura 1).

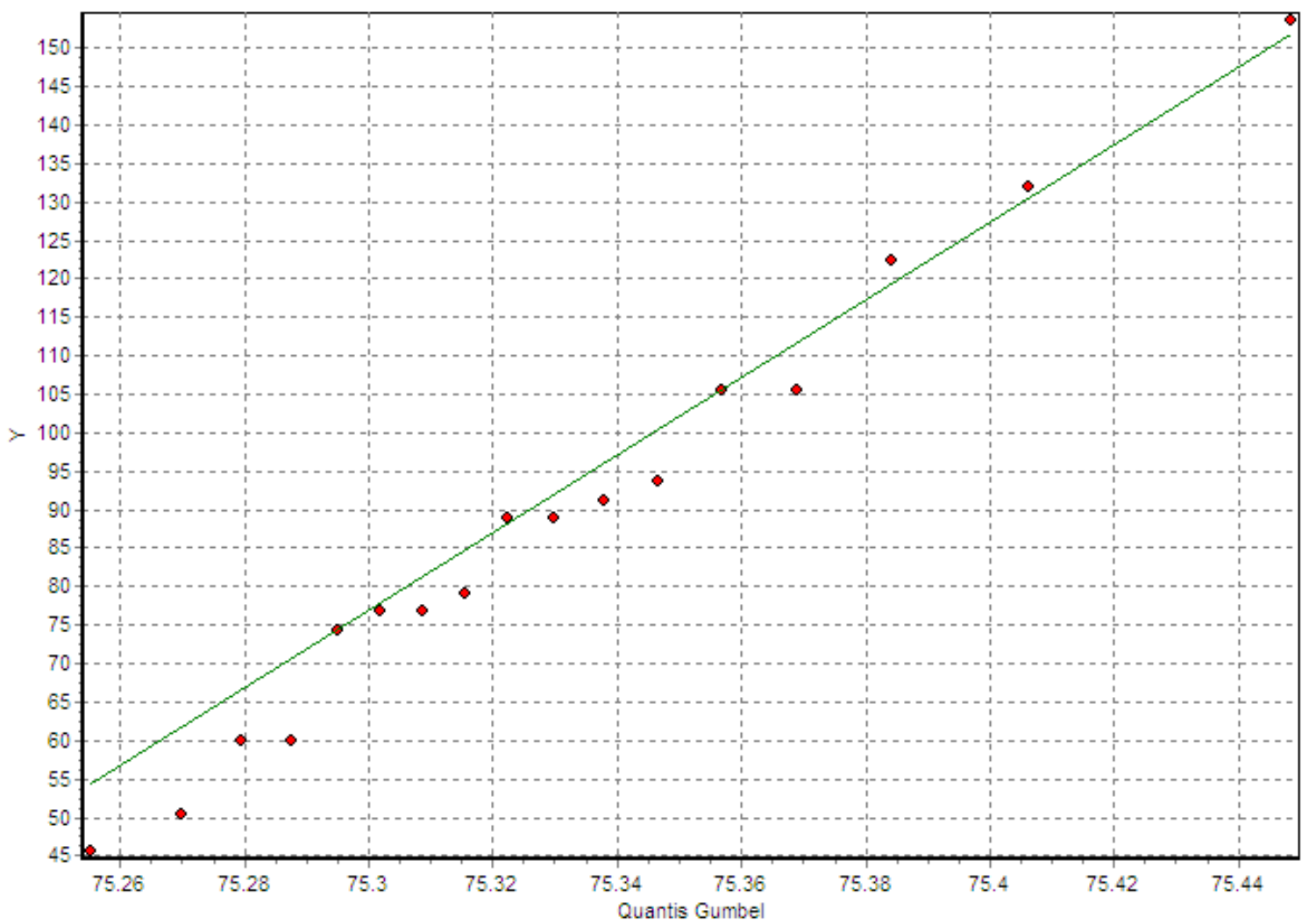

FIGURA 1. Valores de precipitação máxima diária anual observada (Y) e os quantis obtidos pelo modelo Gumbel de probabilidade. Values for the recorded maximum daily precipitation (Y) and the quantiles calculated through Gumbel's probability model. 
A variável reduzida da distribuição de Gumbel foi obtida pela aplicação da função de máxima verossimilhança. Os valores encontrados para os parâmetros da distribuição Gumbel, $\alpha$ representando a escala, e $\mu$ a posição, foram 0,04296 e 75,30677, respectivamente. Assim, aplicando-se a Função Cumulativa de Probabilidade, para os períodos de retorno de 2; 5 e 10 anos, as alturas de lâmina estimadas foram 83,2; 109,3 e 126,5 mm, respectivamente.

$\mathrm{Na}$ Tabela 2, encontram-se os valores de intensidades máximas $\left(\mathrm{mm} \mathrm{h}^{-1}\right)$ obtidos da curva IDF histórica, pelo Método das Relações (CETESB, 1979), BELTRAME et al. (1991), ROBAINA \& PEITER (1992) e pelo modelo Bartlett-Lewis do Pulso Retangular Modificado (DAMÉ, 2001), para as durações e períodos de retorno preestabelecidos.

TABELA 2. Valores de intensidades máximas $\left(\mathrm{mm} \mathrm{h}^{-1}\right)$ obtidos da curva IDF histórica da cidade de Pelotas - RS, pelo Método das Relações (CETESB, 1979), BELTRAME et al. (1991), ROBAINA \& PEITER (1992) e DAMÉ (2001), para as diferentes durações (D) e períodos de retorno (Tr). Values for maximum precipitation intensity $\left(\mathbf{m m ~ h}^{\mathbf{- 1}}\right)$, calculated by the Relationships Method (CETESB, 1979), BELTRAME et al. (1991), ROBAINA \& PEITER (1992) e DAMÉ (2001) for different duration (D) and return periods (Tr), derived from the IDF curve on historical recorded data from the city of Pelotas - RS, Brazil.

\begin{tabular}{|c|c|c|c|c|c|c|}
\hline $\operatorname{Tr}$ & D & Histórica & $\begin{array}{l}\text { Método das Relações } \\
\text { (CETESB, 1979) }\end{array}$ & $\begin{array}{l}\text { BELTRAME } \\
\text { et al. (1991) }\end{array}$ & $\begin{array}{c}\text { ROBAINA \& } \\
\text { PEITER (1992) }\end{array}$ & $\begin{array}{l}\text { DAMÉ } \\
(2001)\end{array}$ \\
\hline (anos) & $(\min )$ & & - & $\left(\mathrm{mm} \mathrm{h}^{-1}\right)$ & ------------------- & \\
\hline \multirow{6}{*}{2} & 15 & 84,4 & 82,5 & 93,2 & 81,6 & 76,0 \\
\hline & 30 & 56,6 & 59,0 & 63,4 & 54,6 & 56,6 \\
\hline & 60 & 37,6 & 39,8 & 38,3 & 34,7 & 38,4 \\
\hline & 360 & 12,4 & 11,4 & 10,7 & 9,4 & 9,9 \\
\hline & 720 & 6,2 & 6,7 & 6,4 & 5,5 & 6,1 \\
\hline & 1.440 & 3,7 & 4,0 & 3,7 & 3,3 & 3,3 \\
\hline \multirow{6}{*}{5} & 15 & 117,9 & 146,5 & 115,6 & 124,1 & 100,5 \\
\hline & 30 & 83,2 & 77,5 & 78,4 & 84,6 & 75,8 \\
\hline & 60 & 55,7 & 52,3 & 49,6 & 54,5 & 61,2 \\
\hline & 360 & 15,5 & 15,0 & 14,2 & 14,9 & 14,5 \\
\hline & 720 & 9,3 & 8,8 & 8,5 & 8,8 & 8,9 \\
\hline & 1.440 & 4,6 & 5,2 & 4,9 & 5,2 & 4,7 \\
\hline \multirow{6}{*}{10} & 15 & 120,0 & 125,5 & 130,0 & 160,5 & 106,2 \\
\hline & 30 & 90,4 & 89,6 & 88,6 & 111,0 & 88,8 \\
\hline & 60 & 60,6 & 60,6 & 57,1 & 72,1 & 75,0 \\
\hline & 360 & 17,7 & 17,3 & 16,5 & 19,8 & 15,2 \\
\hline & 720 & 11,1 & 10,2 & 9,9 & 11,7 & 10,3 \\
\hline & 1.440 & 5,6 & 6,0 & 5,7 & 6,9 & 5,6 \\
\hline
\end{tabular}

Os resultados do teste " $\mathrm{t}$ " aplicados aos coeficientes linear e angular, bem como os respectivos níveis de significância para cada um dos métodos de desagregação estudados são apresentados na Tabela 3.

Observa-se que, para o período de retorno de 2 anos, a hipótese de nulidade foi aceita, visto que não houve diferença significativa, a $5 \%$ de probabilidade, e, portanto, as quatro metodologias estudadas são consideradas adequadas para a obtenção das curvas IDF. Os resultados dos níveis de significância, tanto para $\beta_{0}$ como para $\beta_{1}$, podem ser expressos pela sentença: "a variação entre os valores de intensidades máximas históricos e obtidos mediante os métodos de desagregação de precipitação diária não foi significativa". No entanto, os valores do Erro Relativo Médio Quadrático (RMS), encontrados na Tabela 4, indicam que o método das Relações (CETESB, 1979) e de BELTRAME et al. (1991) foram os que apresentaram maior grau de precisão, comparados aos demais, visto os menores valores percentuais: 6,34 e $8,67 \%$, respectivamente. 
No entanto, o Método das Relações é de mais fácil aplicação do que o de BELTRAME et al. (1991), pois este necessita que se conheça o mapa das isolinhas de precipitações intensas médias para 24 horas, bem como as equações de precipitações intensas dadas em função da duração e do período de retorno. O método das relações necessita apenas que a série de precipitações máximas diárias seja ajustada a uma distribuição teórica de probabilidade e, uma vez essa eleita, aplica-se sobre esse valor os coeficientes de desagregação (CETESB, 1979), que são amplamente conhecidos na bibliografia (Tabela 1).

TABELA 3. Resultados obtidos com o ajuste linear $\left(Y=\beta_{0}+\beta_{1} X\right)$ entre os valores de intensidades máximas $\left(\mathrm{mm} \mathrm{h}^{-1}\right)$ obtidos da curva IDF histórica da cidade de Pelotas - RS, pelo Método das Relações (CETESB, 1979) ${ }^{1}$, BELTRAME et al. $(1991)^{2}$, ROBAINA \& PEITER (1992) ${ }^{3}$ e DAMÉ $(2001)^{4}$, para os períodos de retorno de $2 ; 5$ e 10 anos. Values for the linear fit model $\left(Y=\beta_{0}+\beta_{1} X\right)$ between the maximum precipitation intensity $\left(\mathrm{mm} \mathrm{h}^{-1}\right)$, as calculated through the IDF curve on historical records for the city of Pelotas - RS, and return periods of 2,5 and 10 years. Values for maximum precipitation intensity were obtained by the Method of Relationships (CETESB, 1979), BELTRAME et al. (1991), ROBAINA \& PEITER (1992) and DAMÉ (2001).

\begin{tabular}{cccccccc}
\hline $\begin{array}{c}\text { Tr } \\
\text { (anos) }\end{array}$ & Método & $\beta_{0}$ & $\beta_{1}$ & $\mathrm{t}\left(\beta_{0}\right)$ & $\mathrm{t}\left(\beta_{1}\right)$ & $\begin{array}{c}\text { Significância }(\mathrm{p}) \\
\left(\beta_{0}\right)\end{array}$ & $\begin{array}{c}\text { Significância }(\mathrm{p}) \\
\left(\beta_{1}\right)\end{array}$ \\
\hline \multirow{3}{*}{2} & 1 & 0,6328 & 0,9935 & 0,6360 & 0,0065 & 0,5527 & 0,9950 \\
& 2 & $-1,6293$ & 1,1223 & 1,4503 & 0,1088 & 0,2067 & 0,9175 \\
& 3 & $-1,2836$ & 0,9796 & 1,3097 & 0,0208 & 0,2472 & 0,9842 \\
& 4 & 0,5238 & 0,9316 & 0,5601 & 0,0731 & 0,5995 & 0,9944 \\
\hline \multirow{3}{*}{5} & 1 & $-4,8448$ & 1,1683 & 4,0769 & 0,1416 & 0,0096 & 0,8929 \\
& 2 & $-1,0584$ & 0,9698 & 1,0902 & 0,0311 & 0,3253 & 0,9764 \\
& 3 & $-1,2083$ & 1,0459 & 1,1545 & 0,0438 & 0,3005 & 0,9667 \\
& 4 & 2,8402 & 0,8685 & 3,2432 & 0,1501 & 0,0229 & 0,8865 \\
\hline \multirow{3}{*}{10} & 1 & $-1,0961$ & 1,0340 & 1,0593 & 0,3286 & 0,3379 & 0,9751 \\
& 2 & $-2,6493$ & 1,0599 & 2,4919 & 0,0563 & 0,0550 & 0,9573 \\
& 3 & $-3,5109$ & 1,3198 & 2,6545 & 0,2418 & 0,0450 & 0,8185 \\
\hline
\end{tabular}

$\beta_{0}$ e $\beta_{1}$ : coeficientes linear e angular do ajuste linear; $\mathrm{t}_{\operatorname{tab}(5 \%)}=2,5706$; Probabilidade: $5 \%$.

Considerando o período de retorno de 5 anos, tanto o teste $t\left(\beta_{0}\right)$ como o da significância $\left(\beta_{0}\right)$ indicaram que os métodos das relações (CETESB, 1979) e o utilizado por DAMÉ (2001) apresentaram diferenças significativas a $5 \%$ de probabilidade, entre os valores históricos e sintéticos de intensidades máximas de precipitação. Os valores de RMS encontrados (Tabela 4) confirmam a rejeição de $\mathrm{H}_{0}$. Os resultados encontrados são contraditórios no que se refere ao método das relações para o período de retorno de 5 anos, uma vez que, nesse período, a hipótese de nulidade foi rejeitada, e para 2 e 10 anos foi aceita (Tabela 3).

TABELA 4. Valores de erro relativo médio quadrático (RMS) dos dados de intensidade máxima de precipitação obtidos pelos métodos utilizados, comparados a IDF histórica. Mean square relative error (RMS) from data on maximum precipitation intensity calculated from different methods when compared to IDF historical records.

\begin{tabular}{ccccc}
\hline \multirow{2}{*}{$\begin{array}{c}\operatorname{Tr} \\
\text { anos })\end{array}$} & $\begin{array}{c}\text { Método das Relações } \\
(\text { CETESB, 1979) }\end{array}$ & $\begin{array}{c}\text { BELTRAME et al. } \\
(1991)\end{array}$ & $\begin{array}{c}\text { ROBAINA \& } \\
\text { PEITER (1992) }\end{array}$ & $\begin{array}{c}\text { DAMÉ } \\
(2001)\end{array}$ \\
\cline { 2 - 5 } & \multicolumn{3}{c}{ RMS (\%) } \\
\hline 2 & 6,34 & 8,67 & 12,51 & 10,24 \\
5 & 12,10 & 7,67 & 6,49 & 8,75 \\
10 & 4,89 & 6,81 & 21,48 & 12,59 \\
\hline Média & 7,78 & 7,72 & 13,49 & 10,53 \\
\hline
\end{tabular}


Uma hipótese a ser considerada é que os valores relativos ao teste $\mathrm{t}$ e as RMS são resultados de valores médios das intensidades máximas, considerando as seis durações estabelecidas. No entanto, observando-se a Tabela 2, verifica-se que as maiores diferenças entre os valores de intensidades máximas históricos e sintéticos ocorrem nas menores durações (15; 30 e 60 min), o que pode estar contribuindo para a rejeição da hipótese de nulidade no período de retorno de 5 anos.

As séries geradas pela metodologia de modelagem estocástica de simulação de precipitação, Bartlett-Lewis do Pulso Retangular modificado, e de desagregação de precipitação diária apresentada por DAMÉ (2001), não representaram os valores de intensidades máximas históricos, quando os períodos de retorno foram de 5 e 10 anos.

A simulação da precipitação foi realizada na duração de 15 minutos, e os parâmetros estimados para cada um dos 12 meses do ano, no período de 1982 a 1998; em seis anos ocorreram eventos de "El Niño" (1982, 1983, 1987, 1992, 1997 e 1998). Quando ocorreram esses fenômenos, as alturas $(\mathrm{mm})$ e as intensidades $\left(\mathrm{mm} \mathrm{h}^{-1}\right)$ de precipitação foram maiores que as normais. Além disso, a sua ocorrência foi de alta intensidade em apenas alguns meses do ano. Há que se considerar que os parâmetros do modelo foram estimados pelo método dos momentos, o que corrobora o incremento das incertezas, ao invés do método da máxima verossimilhança, que, embora sendo clássico na estimativa de parâmetros, segundo RODRIGUEZ-ITURBE et al. (1987), não é adequado no caso do modelo BLPRM, pois a função verossimilhança tende a enfatizar a idealização inerente ao modelo, no que diz respeito à trajetória dos eventos em tempo contínuo.

À semelhança de DAMÉ (2001), para o período de retorno de 10 anos, o modelo proposto por ROBAINA \& PEITER (1992) não representou os valores históricos de intensidades máximas de precipitação (Tabela 3), e o desvio em relação a esses foi de 21,48\% (Tabela 4). No trabalho de ROBAINA (1996), utilizando o mesmo modelo, os desvios máximos encontrados entre as precipitações estimadas e as observadas em 32 localidades do Rio Grande do Sul foram em torno de $15 \%$, o que fez com que o autor considerasse o modelo adequado para a estimativa de precipitações máximas anuais médias de duração menor que 24 horas.

\section{CONCLUSÕES}

Para a série de precipitação analisada, o método que representou a amostra de dados de intensidades máximas de precipitação para a localidade de Pelotas - RS, nos períodos de retorno de 2 e 10 anos, foi o Método das Relações (CETESB, 1979), sendo esse, portanto, o recomendado para desagregar a precipitação diária.

Houve ganho de informação em termos de curva IDF quando utilizados os métodos das relações e o proposto por BELTRAME et al. (1991), para os períodos de retorno de 2 e 10 anos.

O método de desagregação de precipitação diária proposto por ROBAINA \& PEITER (1991), para o período de retorno de 10 anos, não apresentou ganho de informação em termos de curvas IDF.

O método das relações possui maior facilidade de aplicação do que o proposto por BELTRAME et al. (1991).

O modelo Bartlett-Lewis do Pulso Retangular modificado e de desagregação de precipitação diária apresentada por DAMÉ (2001) não apresentou ganho de informação em termos de curvas IDF para os períodos de retorno de 5 e 10 anos.

\section{REFERÊNCIAS}

BELTRAME, L.F.S.; LANNA, A.E.L.; LOUZADA, J.A.S. Chuvas intensas. Porto Alegre: IPHUFRGS, 1991. 69 p.

BRUSA, L.C. Aprimoramento estatístico da regionalização de vazões máximas e médias: aplicação a bacias hidrográficas do Rio Grande do Sul e Santa Catarina. 2004. 188 f. Tese (Doutorado em 
Engenharia de Recursos Hídricos e Saneamento Ambiental) - Universidade Federal do Rio Grande do Sul, Porto Alegre, 2004.

CETESB. COMPANHIA DE TECNOLOGIA DE SANEAMENTO AMBIENTAL. Drenagem urbana: manual de projeto. São Paulo, 1979. 476 p.

DAMÉ, R.C.F. Desagregação de precipitação diária para estimativa de curvas intensidadeduração-frequência. 2001. 131 f. Tese (Doutorado em Engenharia de Recursos Hídricos e Saneamento Ambiental) - Instituto de Pesquisas Hidráulicas, Universidade Federal do Rio Grande do Sul, Porto Alegre, 2001.

GLASBEY, C.A.; COOPER, G.; McGECHAN, M.B. Disaggregation of daily rainfall by conditional simulation from a point-process model. Journal of Hydrology, Amsterdam, v.165, n.1-4, p.1-9, 1995.

KOUTSOYIANNIS, D.; XANTHOPOULOS, T. A dynamic model for short-scale rainfall disaggregation. Hydrological Sciences Journal, Oxford, v.35, n.3, p.303-22, 1990.

LANNA, A.E. Elementos de estatística e probabilidades. In: TUCCI, C.E.M. Hidrologia: Ciência e aplicação. Porto Alegre: Ed. Universidade/ABRH, 2001. p.79-164.

MACHADO, A.A.; CONCEIÇÃO, A.R. WinStat: sistema de análise estatística para Windows. Universidade Federal de Pelotas, 2006. Disponível em: www.ufpel.edu.br/ amachado. Acesso: 4 fev. 2007.

OCCHIPINTI, A.G.; SANTOS, P.M. Relações entre as precipitações máximas de "um dia" e de "24 horas" na cidade de São Paulo. São Paulo: IAG/USP, 1966.

PEDROLLO, O.C. GEDAC: Gerenciamento de dados contínuos. Manual do usuário. Versão 1.0. Porto Alegre: IPH-UFRGS, 1997. 58 p.

ROBAINA, A.D. Modelo para geração de chuvas intensas no Rio Grande do Sul. Revista Brasileira de Agrometeorologia, Santa Maria, v. 4, n.2, p.95-8, 1996.

ROBAINA, A.D.; PEITER, M.X. Modelo de desagregação e de geração de chuvas intensas no RS. In: CONGRESSO BRASILEIRO DE ENGENHARIA AGRÍCOLA, 21.; SIMPÓSIO DE ENGENHARIA AGRÍCOLA DO CONE SUL, 1., 1992, Santa Maria. Anais... Santa Maria: Sociedade Brasileira de Engenharia Agrícola, 1992. p.746-53.

RODRIGUEZ-ITURBE, I.; COX, D.R.; ISHAM, V. A point process model for rainfall: further developments. Proceedings of the Royal Society of London, Series A, v.417, p. 283-98, 1987. 